\title{
A Case of Delayed Neurological Recovery with Luxury Perfusion and a High Intracranial Arterial Calcification Burden
}

\author{
Hong-Il Suh, Seon-Wook Lee, Young-In Eom, Jin Soo Lee \\ Department of Neurology, Ajou University Medical Center, Suwon, Korea
}

\section{Dear Sir,}

Although patients with acute ischemic stroke achieve recanalization, approximately $37 \%-65 \%$ of them do not show immediate improvement. ${ }^{1}$ The term "stunned brain syndrome" refers to delayed neurological recovery after an ischemic insult to the brain. ${ }^{1}$ We describe the case of a patient with delayed neurological recovery who had a small cerebral infarction but relatively rapid compensatory perfusion and high calcification burden in the intracranial arteries.

A 71-year-old woman visited our emergency room 1.5 hours after developing acute right limb weakness. The initial neurological examination revealed right hemiplegia (arm and leg, grade 2 on the Medical Research Council grading system) and global aphasia (National Institutes of Health Stroke Scale score, 22). Non-enhanced computed tomography did not reveal a definite ischemic or hemorrhagic lesion, and intravenous recombinant tissue plasminogen activator was subsequently infused. Computed tomography angiography revealed a moderate degree of stenosis in the left middle cerebral artery (Figure 1A) and a high calcification burden in almost all intracranial arteries (Figure 1A, B). We assumed that an occlusion of the middle cerebral artery might be in the process of recanalization. Immediate magnetic resonance imaging revealed a small acute infarction near the posterior pole of the lateral ventricle on diffusion-weighted imaging (Figure 1C). Perfusion magnetic resonance imaging performed simultaneously revealed luxury perfusion in the left posterior temporo-parieto-occipital area (Figure 1D). Although her neurological symptoms had improved
24 hours after onset, she still had right hemiparesis (arm and leg, grade 4) and dense sensory aphasia (National Institutes of Health Stroke Scale score, 8). The localization of these neurological deficits corresponded to the area of luxury perfusion. On the fourth day after admission, the small lesion persisted on diffusion-weighted imaging (Figure 1E), and the luxury perfusion had normalized (Figure 1F), but the neurological deficits including sensory aphasia remained (National Institutes of Health Stroke Scale score, 7). By the sixth day after admission, her neurological deficits had completely disappeared.

The patient showed some immediate neurological recovery, but sensory aphasia persisted even though the diffusion-restricted lesion was small and the cerebral perfusion pattern was compensatory. We propose that this delayed neurological recovery was associated with vascular aging, which was reflected by the high intracranial arterial calcification burden. Calcium deposition in the vasculature is one of the main components of vascular aging, ${ }^{2}$ which is associated with poor adaptation following ischemia/reperfusion injuries. ${ }^{3}$ In addition, neovascularization following reperfusion is hampered by the vascular calcification process. ${ }^{4}$ Reperfusion may result in increased levels of reactive oxygen species that subsequently impair endothelial progenitor cell function. ${ }^{5}$ With regard to post-stroke repair mechanisms, endothelial progenitor cells are known to be essential for neovascularization. ${ }^{6,7}$ The delayed neurological recovery could be explained by these poor adaptations and hampered neovascularization following revascularization.

The delayed neurological recovery in this patient could also have been associated with the luxury perfusion phenomenon; 

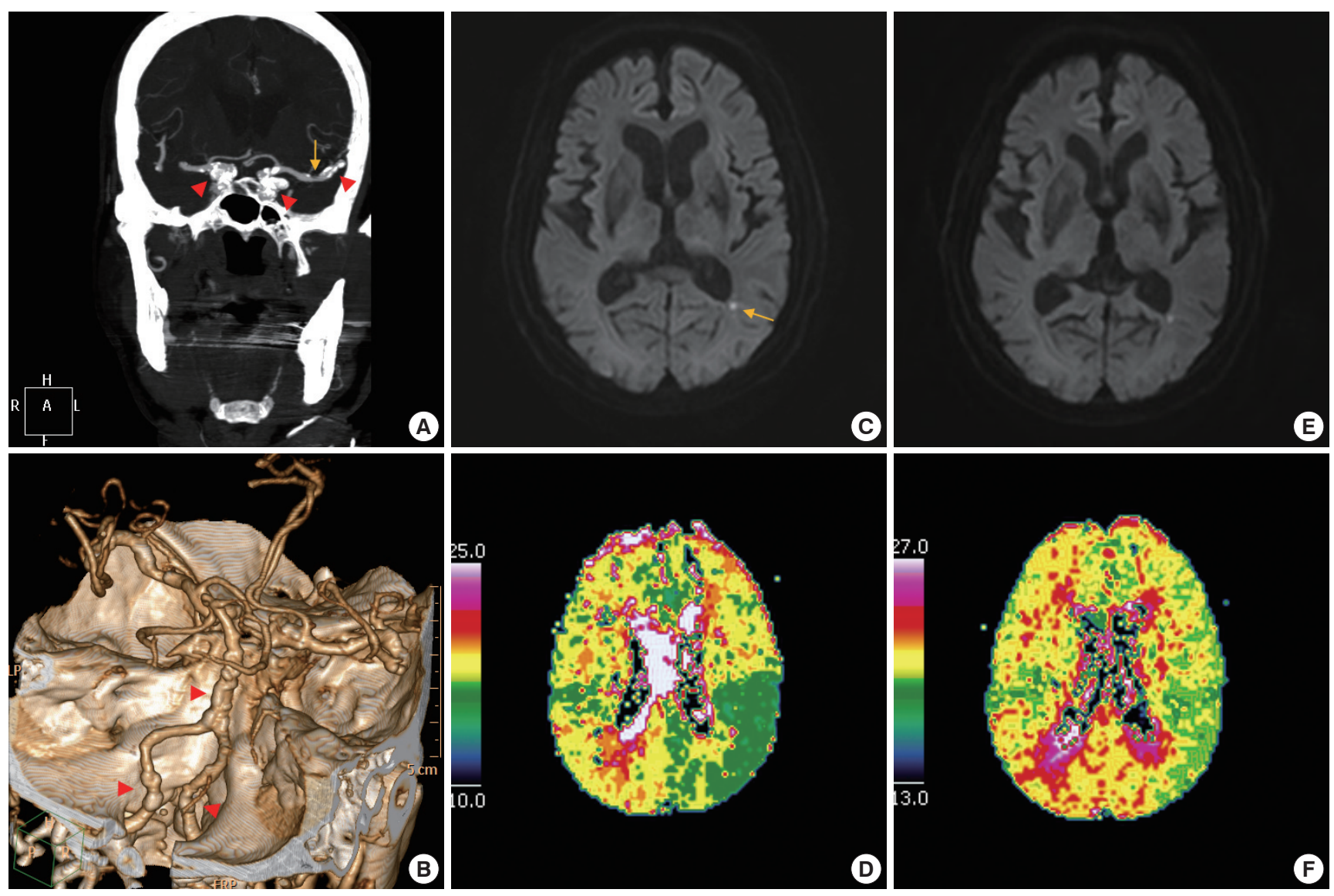

Figure 1. Initial computed tomography (CT) and magnetic resonance (MR) images of the patient in this study. (A) A coronal maximum intensity projection image from CT angiography shows significant stenosis of the distal left middle cerebral artery, which was lined with calcified plaques (arrow). High calcification burden is also seen along the bilateral distal internal carotid arteries (arrowhead). (B) A volume-rendered image from CT angiography shows a high calcification burden along all intracranial arteries (arrowhead). (C) Diffusion-weighted imaging performed on admission demonstrates a small lesion on the left parieto-occipital area (arrow). (D) A time-to-peak map of MR perfusion imaging performed on admission shows extensive luxury perfusion in the posterior temporo-parieto-occipital area. (E) Diffusionweighted imaging demonstrates the persistence of the small lesion on the fourth day after admission. (F) The abnormal finding on the perfusion MR image had normalized by the fourth day after admission.

however, a previous report has shown a relationship with perfusion deficit. Hyperperfusion on perfusion magnetic resonance imaging is thought to reflect a high degree of ischemic stress. ${ }^{8}$ The hyperperfused area was reported to correspond to the area of cerebral infarction in this patient. When compared to patients with normal perfusion, those with hyperperfusion show lower apparent diffusion coefficient values. ${ }^{8}$ Moreover, delayed clinical recovery has been reported to be associated with perfusion deficits. ${ }^{1}$ A defect that extends beyond a diffusion-restricted area is referred to as an ischemic penumbra, ${ }^{9}$ which exhibits deranged cerebral metabolism and can be identified using perfusion imaging. The present case indicates that either hyperperfusion or hypoperfusion may be associated with delayed neurological recovery after cerebral arterial recanalization. ${ }^{8}$

In conclusion, the delayed neurological recovery observed in our patient might have resulted from either post-ischemic reperfusion syndrome associated with luxury perfusion, or an im- paired post-stroke repair mechanism associated with a high burden of intracranial arterial calcification. Computed tomography and magnetic resonance imaging are both useful for estimating the degree of ischemic injury.

\section{Acknowledgements}

This work was supported by the new faculty research fund of the Ajou University School of Medicine.

\section{References}

1. Bang OY, Liebeskind DS, Saver JL, Kim GM, Chung CS, Lee $\mathrm{KH}$. Stunned brain syndrome: serial diffusion perfusion MRI of delayed recovery following revascularisation for acute ischaemic stroke. J Neurol Neurosurg Psychiatry 2011;82:27-32.

2. Lee HY, Oh BH. Aging and arterial stiffness. Circ J 2010;74: 
2257-2262.

3. Palomares SM, Cipolla MJ. Vascular Protection Following Cerebral Ischemia and Reperfusion. J Neurol Neurophysiol 2011; 20:S1-004.

4. Serrano CV Jr, Oranges M, Brunaldi V, de MSA, Torres TA, Nicolau JC, et al. Skeletonized coronary arteries: pathophysiological and clinical aspects of vascular calcification. Vasc Health Risk Manag 2011;7:143-151.

5. Fleissner F, Thum T. Critical role of the nitric oxide/reactive oxygen species balance in endothelial progenitor dysfunction. Antioxid Redox Signal 2011;15:933-948.

6. Asahara T, Murohara T, Sullivan A, Silver M, van der Zee R, Li $\mathrm{T}$, et al. Isolation of putative progenitor endothelial cells for angiogenesis. Science 1997;275:964-967.

7. Font MA, Arboix A, Krupinski J. Angiogenesis, neurogenesis and neuroplasticity in ischemic stroke. Curr Cardiol Rev 2010; 6:238-244.
8. Kidwell CS, Saver JL, Mattiello J, Starkman S, Vinuela F, Duckwiler G, et al. Diffusion-perfusion MRI characterization of postrecanalizationhyperperfusion in humans. Neurology 2001;57: 2015-2021.

9. Lee DH, Kang DW, Ahn JS, Choi CG, Kim SJ, Suh DC. Imaging of the ischemic penumbra in acute stroke. Korean J Radiol 2005;6:64-74.

\footnotetext{
Correspondence: Jin Soo Lee Department of Neurology, Ajou University Medical Center, 164 Worldcup-ro, Yeongtong-gu, Suwon 443-380, Korea

Tel: +82-31-219-5175, Fax: +82-31-219-5178

E-mail: jinsoo22@gmail.com

Received: November 18, 2013

Revised: January 11, 2014

Accepted: January 11, 2014

The authors have no financial conflicts of interest.
} 